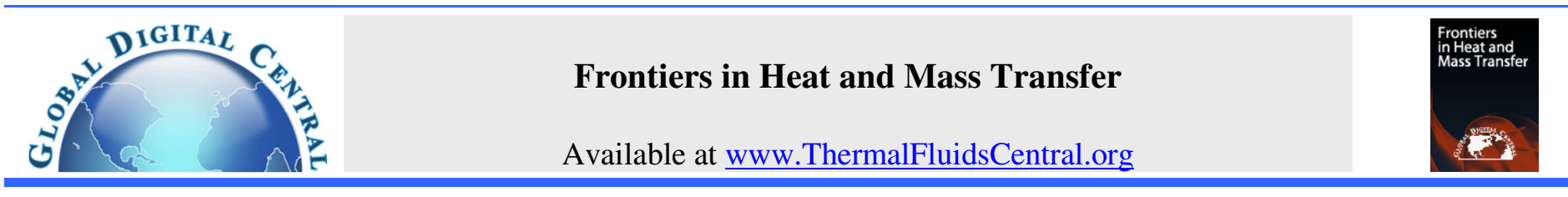

\title{
FORCED CONVECTION BOUNDARY LAYER STAGNATION-POINT FLOW IN DARCY-FORCHHEIMER POROUS MEDIUM PAST A SHRINKING SHEET
}

\author{
Shahirah Abu Bakar ${ }^{\mathrm{a}}$, Norihan Md. Arifin ${ }^{\mathrm{a}, * *}$, Roslinda Nazar $^{\mathrm{b}}$, Fadzilah Md. Ali $^{\mathrm{a}}$, Ioan Pop ${ }^{\mathrm{c}}$ \\ ${ }^{a}$ Department of Mathematics \& Institute For Mathematical Research, Universiti Putra Malaysia, 43400 UPM Serdang, Selangor, Malaysia. \\ ${ }^{b}$ Faculty of Science and Technology, Universiti Kebangsaan Malaysia, 43600 UKM Bangi, Selangor, Malaysia. \\ ${ }^{c}$ Department of Mathematics, Babes-Bolyai University, R-400084 Cluj-Napoca, Romania
}

\begin{abstract}
A mathematical model of forced convection boundary layer stagnation-point slip flow in Darcy-Forchheimer porous medium over a shrinking sheet is presentedin this paper. The governing partial differential equations are transformed into ordinary differential equation using self-similarity transformation which are then solved numerically with shooting method. A parametric study of the physical parameters involved in the problem is conducted and representative set of numerical results are presented through graphs and tables, and are discussed.

Keywords: boundary layer, heat transfer, shrinking, stagnation-point flow, slip effects.
\end{abstract}

\section{INTRODUCTION}

The analysis of convection boundary layer flow along a vertical surface embedded in porous media has received considerable theoretical and practical interest. The convection boundary layer flow occurs in several industrial and technical applications such as electro-chemistry, solar collectors and polymer processing. Studies of boundary layer flows in a saturated porous medium have been considered by several authors. The first study of the free convection flow over a horizontal flat plate embedded in a porous medium was reported by Cheng and Chang (1976) and followed by Cheng (1977) for the mixed convection flow case. Hong et al. (1987) studied the Darcy-Brinkman forced convection flow over a fixed impermeable heated plate embedded in a porous medium. Mukhopadhyay and Layek (2009) analyzed the forced convection boundary layer flow over a porous plate in porous media with the radiation effects. Mahdy and Chamkha (2010) reported the effects of chemical reaction and viscous dissipation on Darcy-Forchheimer mixed convection along a vertical surface in a fluid saturated porous media. The steady forced convection flow and heat transfer past a porous plate placed in a fluid saturated porous medium using the Darcy model with partial slip was discussed by Bhattacharyya et al. (2011). Later, Mukhopapadhyay et al. (2012) have studied the steady forced convection boundary layer flow past a porous plate placed in a fluid-saturated porous medium using the Darcy-Forchheimer model taking into account the effect of thermal radiation. Bakar et al. (2014) considered the forced convection flow past a permeable plate embedded in a DarcyForchheimer poeous medium.

Recently, the boundary layer flow due to a shrinking sheet has attracted considerable interest where there are plenty of applications of shrinking sheet problems in industries and engineering fields. The shrinking sheet occurs, for example, on a rising shrinking balloon. Fang et al. (2010) investigated analytically the viscous flow over a shrinking sheet with a second slip flow model. Lok et al. (2011) studied the MHD Stangnation-Point Flow towards a Shrinking Sheet. Stability of dual solutions in stagnation-point flow and heat transfer over a porous shrinking sheet with thermal radiation was analyzed by Mahapatra and Nandy (2013). Later, Mahapatra and Nandy (2013) presented the unsteady boundary layer flow and heat transfer about a stagnation-point towards a shrinking sheet in the presence of velocity and thermal slip. They show that multiple solution exits for certain range of the ratio of shrinking velocity which again depend on the unsteadiness parameter and velocity slip parameter. Bhattacharrya et al. (2013) studied the slip effects on the boundary-layer flow of a Casson fluid over a porous stretching/shrinking sheet. Very recently, the similarity equations for steady boundary layer flow and heat transfer over a permeable stretching or shrinking surface embedded in a porous medium using Brinkman model was analyzed by Merkin et al. (2014).

In view of above literature, we study the slip effects on on forced convection boundary layer near the stagnation-point over a shrinking sheet in Darcy-Forchheimer porous medium. The slip condition is taken into accaount in terms of the shear stress. Using similarity variables, a third order and a second order differential equations corresponding to the momentum and thermal boundary layer equations are obtained. These nonlinear equations along with appropriate boundary conditions are then solved numerically using shooting method.

\section{BASIQ EQUATIONS}

We consider the steady two-dimensional boundary layer flow and heat transfer near a stagnation-point towards a shrinking sheet in a porous medium with velocity and thermal slips. The differential equations of fluid motion is based on Forchheimer which accounts for the drag exerted by the porous media, in the study of porous media flow analysis. The governing boundary layer equations for momentum and energy under Boussinesq's approximation are:

$\frac{\partial u}{\partial x}+\frac{\partial v}{\partial y}=0$

* Correspondence Author. Email: norihanarifin@yahoo.com 
$u \frac{\partial u}{\partial x}+v \frac{\partial u}{\partial y}=U_{e} \frac{d U_{e}}{d x}+v \frac{\partial^{2} u}{\partial y^{2}}-\frac{v \varepsilon}{k}\left(u-U_{e}\right)-\frac{F \varepsilon^{2}}{\sqrt{k}}\left(u^{2}-U_{e}^{2}\right)$,

$u \frac{\partial T}{\partial x}+v \frac{\partial T}{\partial y}=\frac{\kappa}{\rho C_{p}} \frac{\partial^{2} T}{\partial y^{2}}$

where $u$ and $v$ are the velocity components along $x$ and $y$, respectively, $v$ is kinematic viscosity, $\mathcal{E}$ is dimensionless stretching/shrinking parameter, $U_{e}=a x$ is the straining velocity of the stagnation-point flow, $k$ is the permeability of the porous media, $T$ is the temperature, $\kappa$ is the thermal conductivity of the fluid and $C_{p}$ is the specific heat at constant pressure. The last two-terms on the right hand side in Eq (2) describe the non-linear Darcy-Forchheimer resistance of the surrounding porous medium. The boundary conditions with partial slip for both velocity and temperature components are given by:

$u=c x+L_{1} \frac{\partial u}{\partial y}, v=0, T=T_{w}+D_{1} \frac{\partial T}{\partial y}$ at $y=0$

$u \rightarrow U_{e}=a x, T \rightarrow T_{\infty}$ as $y \rightarrow \infty$,

where $c$ and $a$ are the shrinking/stretching rate and straining rate parameters, respectively, with $c>0$ is for stretching and $c<0$ is for shrinking. Here $L_{1}$ is velocity slip factor, $D_{1}$ is thermal slip factor, $T_{w}$ is temperature of the sheet and $T_{\infty}$ is free stream temperature.

Next, we introduce the following similarity transformations as follow:

$\psi=\sqrt{a v} x f(\eta), T=T_{\infty}+\left(T_{w}-T_{\infty}\right) \theta(\eta), \eta=y \sqrt{\frac{a}{v}}$.

By using Eq (5), the momentum and temperature in Eq (2) and (3) will obtained as:

$\frac{\partial \psi}{\partial y} \frac{\partial^{2} \psi}{\partial x \partial y}-\frac{\partial \psi}{\partial x} \frac{\partial^{2} \psi}{\partial y^{2}}=U_{e} \frac{d U_{e}}{d x}+v \frac{\partial^{3} \psi}{\partial y^{3}}-\frac{v \varepsilon}{k}\left(\frac{\partial \psi}{\partial y}-U_{e}\right)-$

$\frac{F \varepsilon^{2}}{\sqrt{k}}\left[\left(\frac{\partial \psi}{\partial y}\right)^{2}-U_{e}^{2}\right]$

$\frac{\partial \psi}{\partial y} \frac{\partial T}{\partial x}-\frac{\partial \psi}{\partial x} \frac{\partial T}{\partial y}=\frac{\kappa}{\rho C_{p}} \frac{\partial^{2} T}{\partial y^{2}}$

where $\psi$ is the stream function, which is defined in the usual way as $u=\frac{\partial \psi}{\partial y}$ and $v=-\frac{\partial \psi}{\partial x}$.

The boundary conditions in Eq (4) then reduce to:

$\frac{\partial \psi}{\partial y}=c x+L_{1} \frac{\partial^{2} \psi}{\partial y^{2}}, \frac{\partial \psi}{\partial x}=0, T=T_{w}+D_{1} \frac{\partial T}{\partial y}$ at $y=0$.

By substitute Eq (5) into Eqs (6) and (7), we obtain the following selfsimilar equations:

$f^{\prime \prime \prime}+f f^{\prime \prime}-\left(f^{\prime}\right)^{2}-k_{1}\left(f^{\prime}-1\right)-k_{2}\left[\left(f^{\prime}\right)^{2}-1\right]+1=0$,

$\theta^{\prime \prime}+\operatorname{Pr} f \theta^{\prime}=0$,

where $k_{1}=\frac{v \varepsilon}{k a}$ is the parameter of porous media, $k_{2}=\frac{F \varepsilon^{2} x}{\sqrt{k}}$ is the inertia-coefficient parameter and Pr is the Prandtl number. The boundary conditions finally become:

$$
\begin{aligned}
& f(\eta)=0, f^{\prime}(\eta)=\alpha+\delta f^{\prime \prime}(\eta), \theta(\eta)=1+\beta \theta^{\prime}(\eta) \text { at } \eta=0 \\
& f^{\prime}(\eta) \rightarrow 1, \theta(\eta) \rightarrow 0 \text { as } \eta \rightarrow \infty
\end{aligned}
$$

where $\alpha=\frac{c}{a}$ is the velocity ratio parameter, $\delta=L \sqrt{\frac{a}{v}}$ is velocity slip parameter and $\beta=D \sqrt{\frac{a}{v}}$ is thermal slip parameter.

\section{RESULTS AND DISCUSSION}

The nonlinear ordinary differential equations (9) and (10) subject to the boundary conditions (11) are solved numerically using the Shooting method. To validate the accuracy of the numerical method, we compare the obtained results corresponding to the skin-friction coefficient $f^{\prime \prime}(0)$ with the published results of Bhattacharyya et al. (2011) and Mahapatra and Nandy (2013) in Table 1 and those are found in excellent agreement. This study confirms that the existence and uniqueness of solution depend on the velocity ratio parameter $\alpha$. For the current work, the numerical computations are performed for various values of different parameters such as the parameter of the porous medium $k_{1}$, inertial parameter $k_{2}$, velocity slip parameter $\delta$, thermal slip parameter $\beta$, Prandtl number $\operatorname{Pr}$, and the velocity ratio parameter $\alpha$. Variation of the skin friction coefficient $f^{\prime \prime}(0)$, and temperature gradient $-\theta^{\prime}(0)$ for different values of velocity ratio parameter $\alpha$ and velocity slip parameter $\delta$ are presented in Table 2. It is noticed that the values of $f^{\prime \prime}(0)$ and $-\theta^{\prime}(0)$ increases with the increase of velocity slip parameter $\delta$ that the velocity slip delays the separation of boundary layer. On the other hand, variation of the temperature gradient $-\theta^{\prime}(0)$ with $\alpha$ for different values of $\beta$ when $k_{1}=0.1, k_{2}=0.1, \delta=1.0$ and $\operatorname{Pr}=0.7$ are presented in Table 4 and Figure 3. The first solution is stable, while the second solution is not, according to the stability analysis performed by Weidman et al. (2006) i.e. first solution is physically realizable (stable), while the second solution is not physically realizable (unstable). Also, this investigation confirms that the existence and uniqueness of the solutions greatly depend on the stretching/shrinking velocity parameter $\epsilon$ as also pointed out by Miklavci ${ }^{2} \mathrm{c}$ and Wang (2006). Figures 4 and 5 display the velocity and temperature profiles for several values of $\alpha$ when $\alpha$ when $k_{1}=0.1 k_{2}=0.1, \delta=1.0, \beta=0.1$ and $\operatorname{Pr}=1.0$. The dual velocity profiles show that the velocity decreases with increasing magnitude of $\alpha$ in first solution and conversely for second solution it increases. In Figure 5 , it is observed that the thermal boundary layer thickness for second solution is thicker than the thickness for first solution. The effects

Table 1 Values of skin friction coefficient $f^{\prime \prime}(0)$ for several values of $\alpha$ when $k_{1}=k_{2}=\delta=\beta=0.0$.

\begin{tabular}{|c|c|c|c|}
\hline$\alpha$ & $\begin{array}{c}|c| \\
\text { Bhattacharyya } \\
\text { et al. } \\
(2011)\end{array}$ & $\begin{array}{c}\text { Mahapatra and } \\
\text { Nandy } \\
(2013)\end{array}$ & Present work \\
\hline-0.25 & 1.4022405 & 1.402242 & 1.40224083 \\
\hline-0.5 & 1.4956697 & 1.495672 & 1.49566981 \\
\hline-0.75 & 1.4892981 & 1.489296 & 1.48929830 \\
\hline-1.00 & 1.3288169 & 1.328819 & 1.32881699 \\
\hline-1.15 & 1.0822316 & 1.082232 & 1.08223143 \\
& $(0.1167023)$ & $(0.116702)$ & $(0.11670217)$ \\
\hline-1.20 & 0.9324728 & 0.932470 & 0.93247382 \\
& $(0.2336491)$ & $(0.233648)$ & $(0.23364968)$ \\
\hline-1.246 & 0.5842915 & 0.584374 & 0.58429402 \\
& $(0.5542856)$ & $(0.554215)$ & $(0.55428441)$ \\
\hline- & 0.5745268 & - & 0.57456027 \\
1.24657 & $(0.5639987)$ & & $(0.56397856)$ \\
\hline
\end{tabular}

Results in parenthesis ( ) are the second (dual) solutions 
Table 2 Values of $f^{\prime \prime}(0)$ and $-\theta^{\prime}(0)$ for various values of $\alpha$ and $\delta$ when, $k_{1}=0.1, k_{2}=0.1, \beta=0.1$ and $\operatorname{Pr}=1.0$.

\begin{tabular}{|c|c|c|c|}
\hline$\delta$ & $\alpha$ & $f^{\prime \prime}(0)$ & $-\theta^{\prime}(0)$ \\
\hline \multirow{4}{*}{0.0} & -1.00 & 1.328817 & 0.228327 \\
\cline { 2 - 4 } & -1.10 & 1.186680 & 0.219965 \\
& & $(0.073419)$ & $(0.000336)$ \\
\cline { 2 - 4 } & -1.20 & 0.932474 & 0.211283 \\
& & $(0.233650)$ & $(0.010897)$ \\
\hline \multirow{4}{*}{0.5} & -1.00 & 1.458073 & 0.468428 \\
\cline { 2 - 4 } & -1.10 & 1.484336 & 0.450445 \\
& & $(0.006833)$ & $(0.000094)$ \\
\cline { 2 - 4 } & -1.20 & 1.516508 & 0.426762 \\
& & $(0.147207)$ & $(0.001752)$ \\
\hline \multirow{5}{*}{1.0} & -1.00 & 1.132807 & 0.571321 \\
\cline { 2 - 4 } & -1.10 & 1.181250 & 0.559767 \\
& & $(0.000671)$ & $(0.000052)$ \\
\cline { 2 - 4 } & -1.20 & 1.228366 & 0.547674 \\
& & $(0.079355)$ & $(0.000516)$ \\
\hline \multirow{4}{*}{1.5} & -1.00 & 0.901135 & 0.618026 \\
\cline { 2 - 4 } & -1.10 & 0.943134 & 0.610379 \\
& & $(0.000128)$ & $(0.000013)$ \\
\cline { 2 - 4 } & -1.20 & 0.984705 & 0.602501 \\
& & $(0.0648721)$ & $(0.000232)$ \\
\hline
\end{tabular}

Results in parenthesis ( ) are the second (dual) solutions.

Table 3. Values of $\alpha_{c}, f^{\prime \prime}(0)$ and $-\theta^{\prime}(0)$ for various values of $\delta$ when $k_{1}=0.1, k_{2}=0.1, \beta=0.1$ and $\operatorname{Pr}=1.0$

\begin{tabular}{|c|c|c|}
\hline $\boldsymbol{\delta}$ & $\boldsymbol{\alpha}_{\boldsymbol{c}}$ & $f^{\prime \prime}(0)$ \\
\hline 0.0 & -1.246579 & 0.570892 \\
\hline 0.5 & -1.752691 & 1.189160 \\
\hline 1.0 & -2.408533 & 1.395784 \\
\hline 1.5 & -3.127016 & 1.468032 \\
\hline
\end{tabular}

Table 4. Values of $\alpha_{c}$ and $-\theta^{\prime}(0)$ for various values of $\beta$ when $k_{1}=0.1, k_{2}=0.1, \delta=1.0$ and $\operatorname{Pr}=0.7$.

\begin{tabular}{|c|c|c|}
\hline $\boldsymbol{\beta}$ & $\boldsymbol{\alpha}_{\boldsymbol{c}}$ & $-\theta^{\prime}(0)$ \\
\hline 0.0 & -2.330128 & 0.238037 \\
\hline 0.5 & -2.330128 & 0.212377 \\
\hline 1.0 & -2.330128 & 0.192270 \\
\hline
\end{tabular}

of the velocity slip $\delta$ on the velocity and temperature profiles are presented in Figure 6 and 7, respectively. It is observed that $f^{\prime}(\eta)$

decreases on increasing $\delta$. This is because when slip occurs, fluid velocity near the sheet is no longer equal to the velocity of the shrinking sheet and consequently fluid velocity decreases on increasing velocity slip factor. This indicates that velocity slip factor has a substantial effect on the fluid flow.

From Figure 7, it is noticed that fluid temperature $\theta(\eta)$ increases on increasing $\delta$. Thus, there is an enhancement in the fluid temperature throughout the boundary layer region with the increase in velocity slip parameter. The thermal slip parameter $\beta$ also effects on the fluid temperature, which can be seen in from Figure 8 . It is found that the fluid temperature $\theta(\eta)$ decreases on increasing $\beta$ and this is happened if thermal slip parameter increases, less heat is transferred to the fluid from the sheet and, therefore, temperature is found to decrease.

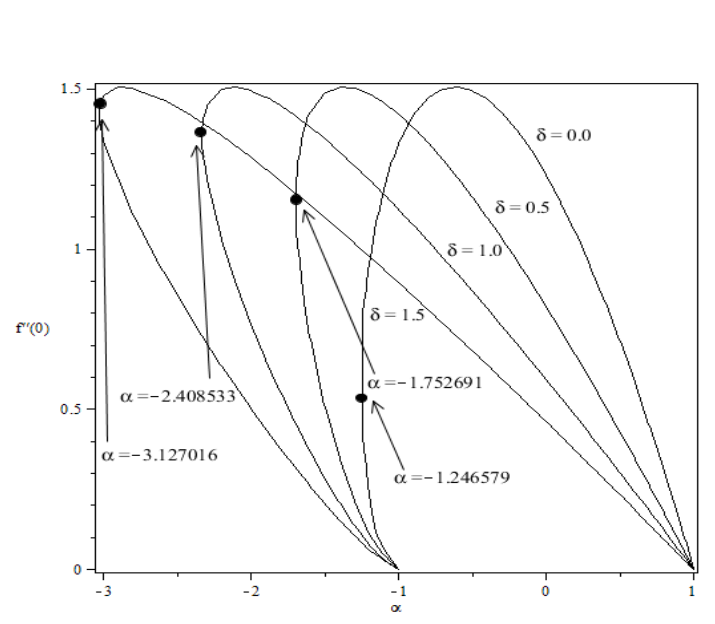

Fig. 1 Variation of skin friction of $f^{\prime \prime}(0)$ with $\alpha$ for several values of $\delta$ when $k_{1}=0.1, k_{2}=0.1, \beta=0.1$ and $\operatorname{Pr}=1.0$.

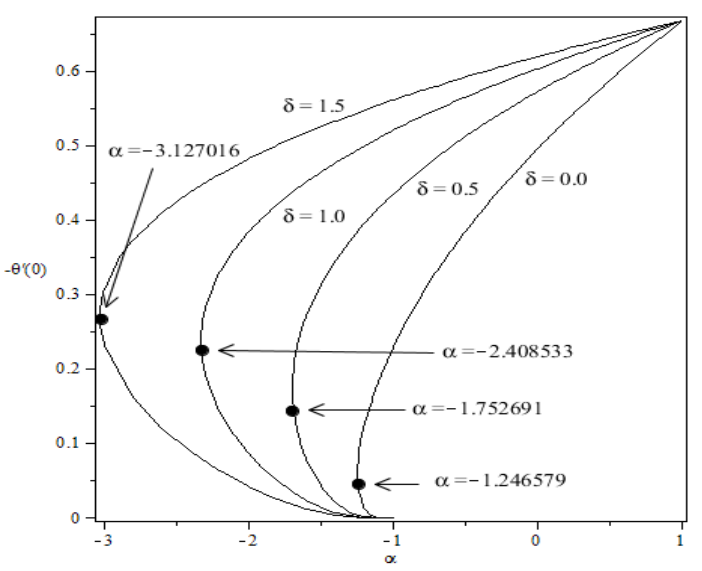

Fig. 2 Variation of temperature gradient $-\theta^{\prime}(0)$ with $\alpha$ for several values of $\delta$ when $k_{1}=0.1, k_{2}=0.1, \beta=0.1$ and $\operatorname{Pr}=1.0$.

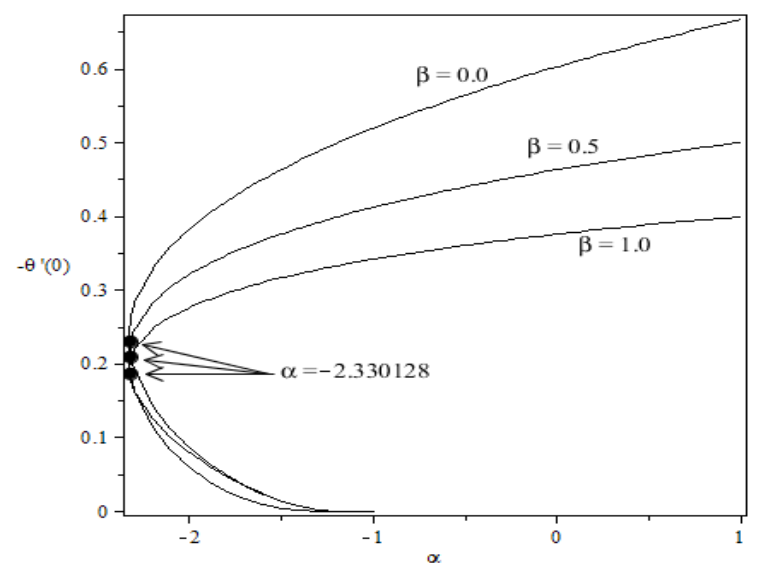

FIG. 3 Variation of temperature gradient $-\theta^{\prime}(0)$ with $\alpha$ for several values of $\beta$ when $k_{1}=0.1, k_{2}=0.1, \delta=1.0$ and $\operatorname{Pr}=0.7$. 


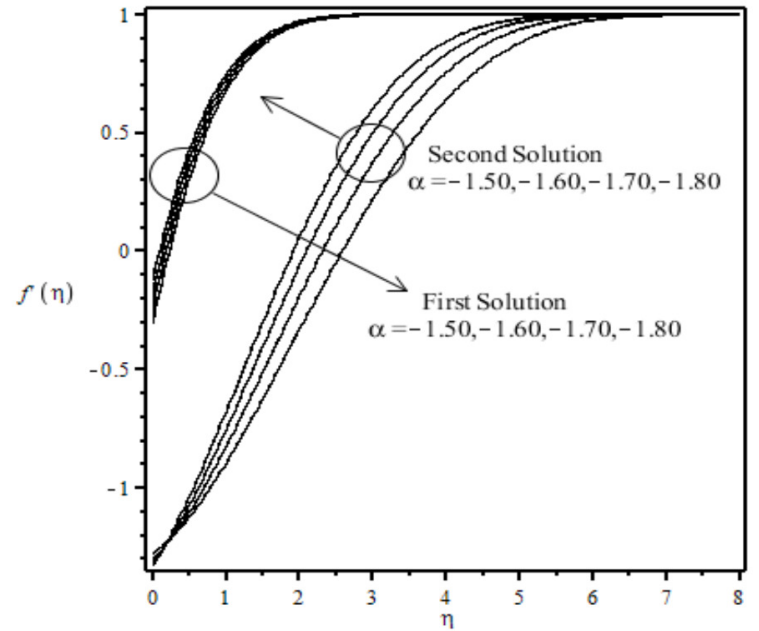

Fig. 4 Velocity profiles $f^{\prime}(\eta)$ for different values of $\alpha$ when $k_{1}=0.1, k_{2}=0.1, \delta=1.0, \beta=0.1$ and $\operatorname{Pr}=1.0$.

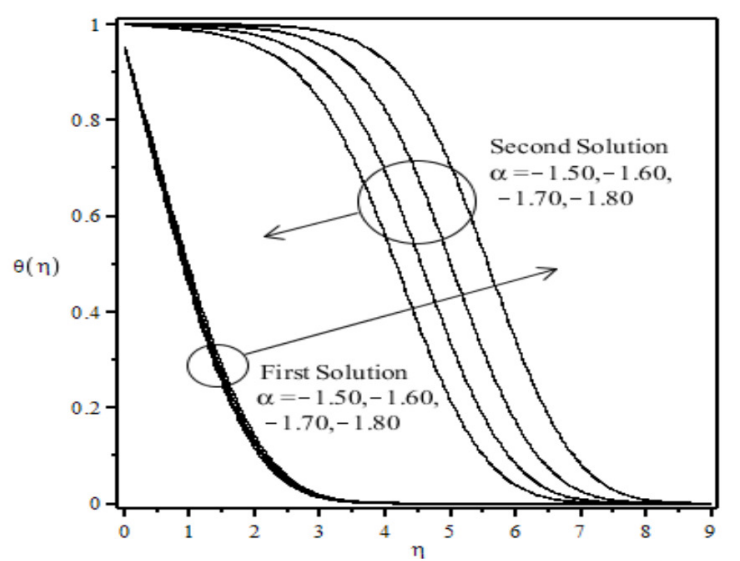

Fig. 5 Temperature profiles $\theta(\eta)$ for different values of $\alpha$ when $k_{1}=0.1, k_{2}=0.1, \delta=1.0, \beta=0.1$ and $\operatorname{Pr}=1.0$.

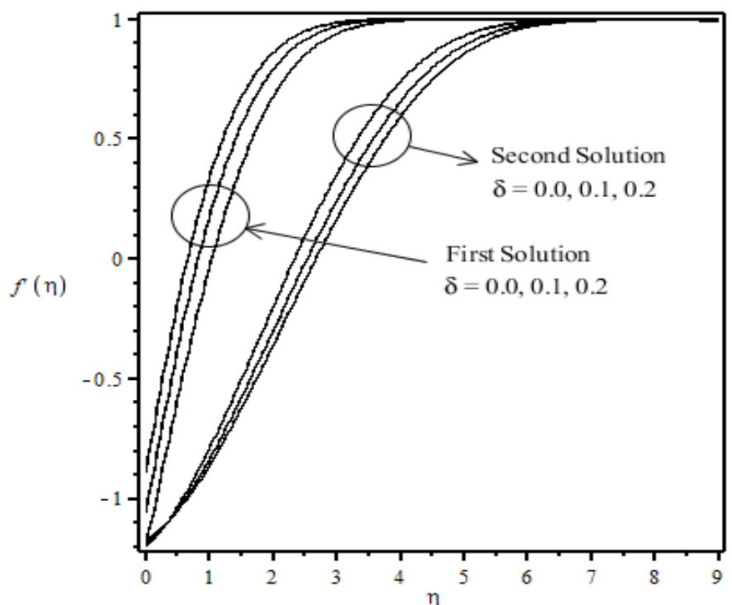

Fig. 6 Velocity profiles $f^{\prime}(\eta)$ for different values of $\delta$ when $k_{1}=0.1, k_{2}=0.1, \alpha=-1.20, \beta=0.1$ and $\operatorname{Pr}=1.0$.

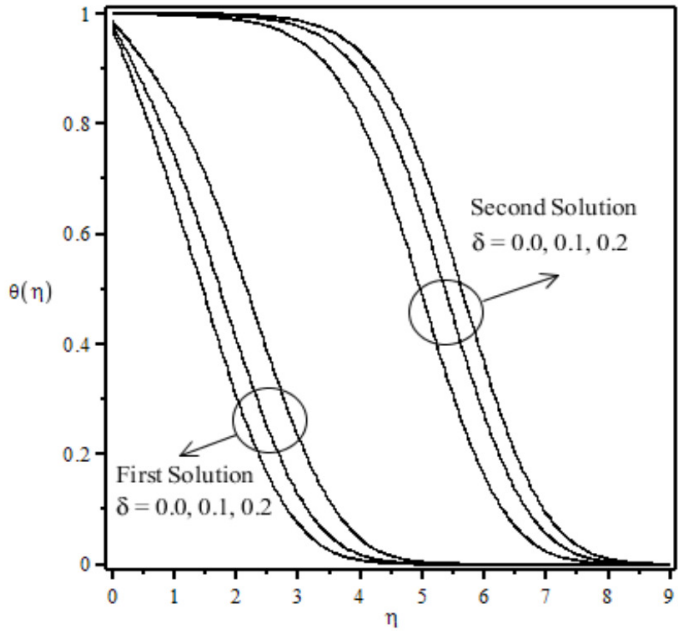

Fig. 7 Temperature profiles $\theta(\eta)$ for different values of $\delta$ when $k_{1}=0.1, k_{2}=0.1, \alpha=-1.20, \beta=0.1$ and $\operatorname{Pr}=1.0$.

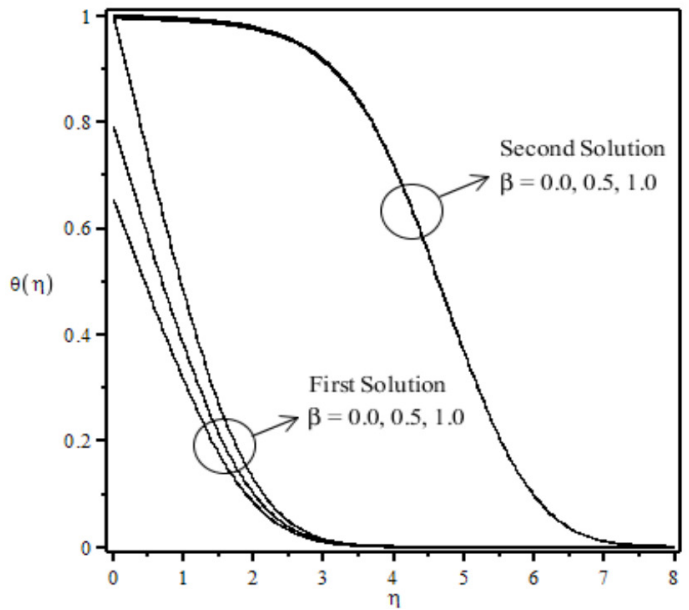

Fig. 8 Temperature profiles $\theta(\eta)$ for different values of $\beta$ when $k_{1}=0.1, k_{2}=0.1, \alpha=-1.50, \delta=1.0$ and $\operatorname{Pr}=1.0$.

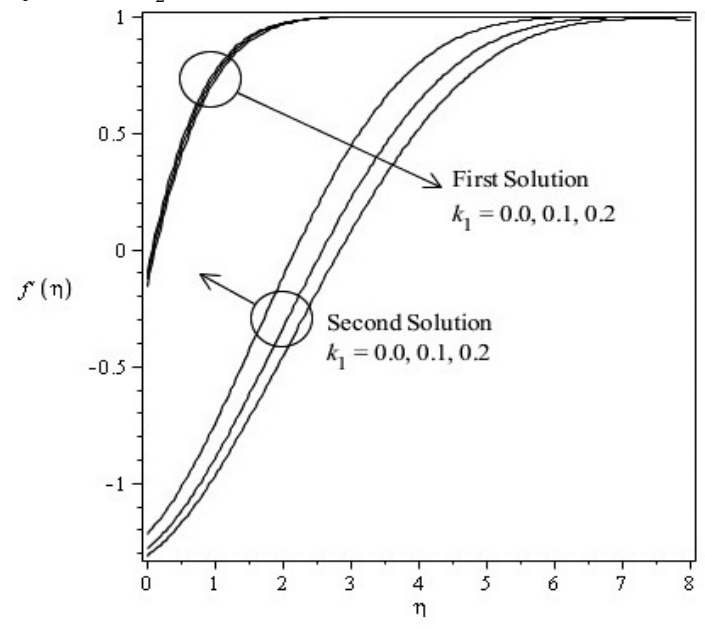

Fig. 9 Velocity profile $f^{\prime}(\eta)$ for different values of $k_{1}$ when $k_{2}=0.1, \alpha=-1.50, \delta=1.0, \beta=0.1$ and $\operatorname{Pr}=0.7$. 


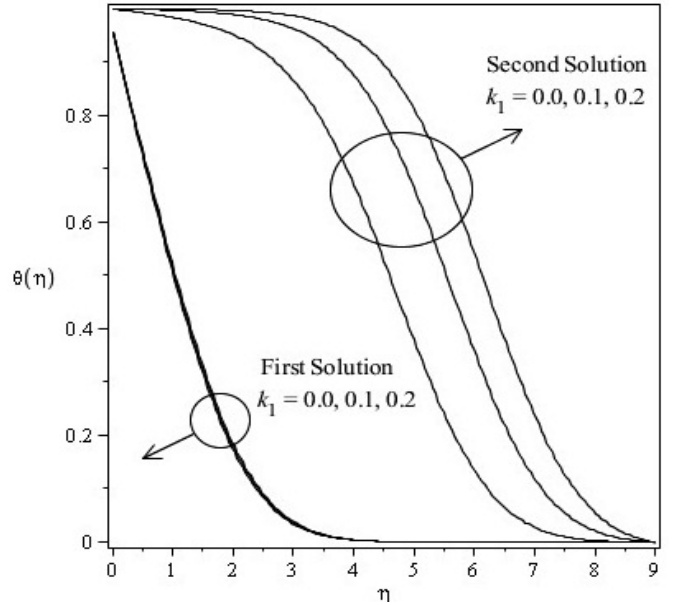

Fig. 10 Temperature profile $\theta(\eta)$ for different values of $k_{1}$ when $k_{2}=0.1, \alpha=-1.50, \delta=1.0, \beta=0.1$ and $\operatorname{Pr}=0.7$.

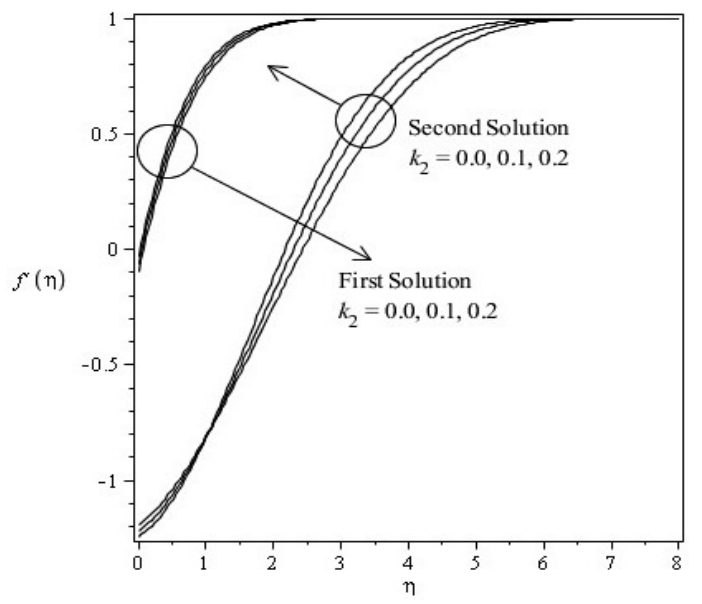

Fig. 11 Velocity profile $f^{\prime}(\eta)$ for different values of $k_{2}$ when $k_{1}=0.1, \alpha=-1.50, \delta=1.0, \beta=0.1$ and $\operatorname{Pr}=0.7$.

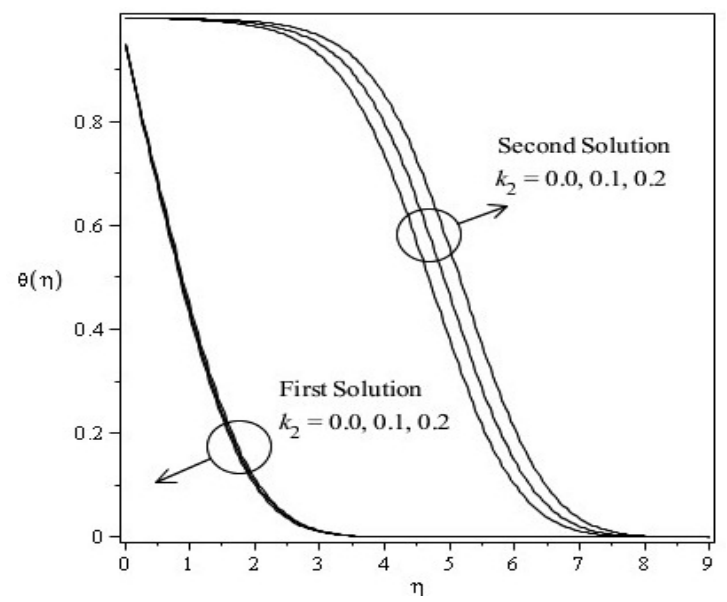

Fig. 12 Temperature profile $\theta(\eta)$ for different values of $k_{2}$ when $k_{1}=0.1, \alpha=-1.50, \delta=1.0, \beta=0.1$ and $\operatorname{Pr}=0.7$.

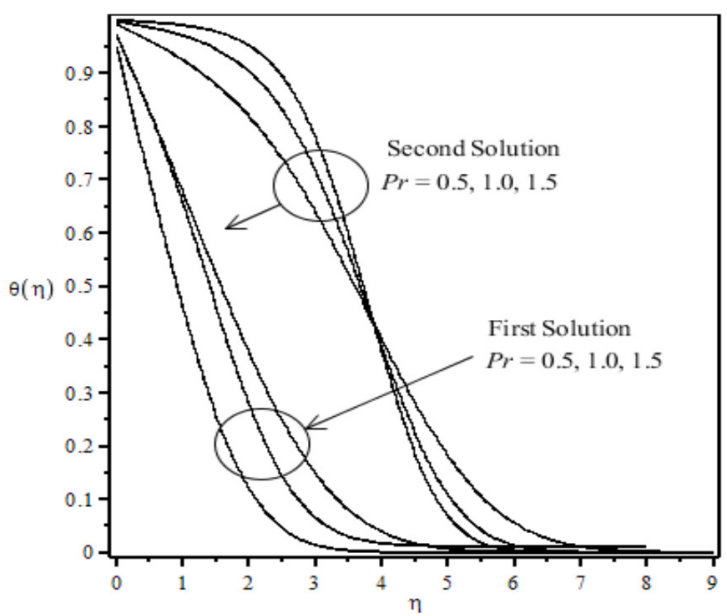

Fig. 13 Temperature profile $\theta(\eta)$ for different values of $\operatorname{Pr}$ when

$$
k_{1}=0.1, k_{2}=0.1, \alpha=-1.50, \delta=0.5 \text { and } \beta=0.1 \text {. }
$$

Thus, there is a reduction in the fluid temperature throughout the boundary layer region. The effect of porous medium parameter $k_{1}$, on velocity and temperature profiles while other parameters are kept constant are illustrated in Figure 9 and 10, respectively. With the increasing values of $k_{1}$, both velocity and temperature profiles show decreases in first solution but conversely increasing in second solution. The influence of inertial parameter $k_{2}$, on velocity and temperature distribution is enlightened in Figure 11 and 12, respectively. For velocity distribution, the velocity decreases as $k_{2}$ is increasing in first solution, while in the second solution case it behaves oppositely. The influence of Prandtl number on temperature distribution is enlightened in Figure 13. As can be seen from this figure, the increasing value of $\mathrm{Pr}$ number causes the decreasing in temperature as well as in boundary layer thickness for both solutions. Physically, when the Prandtl number is small, then the free stream velocity remains continues throughout the walland the velocity profile will be closer to the wall. These results are important for those fluids, whose have a high range of Prandtl number.

\section{CONCLUSIONS}

The forced convection boundary layer stagnation-point flow and heat transfer over a shrinking sheet in Darcy-Forchheimer porous media model with presence of slip is analyzed and discussed in this present paper. The similarity equations are solved numerically and the effects of the parameter of the porous medium $k_{1}$, inertial parameter $k_{2}$, velocity slip parameter $\delta$, thermal slip parameter $\beta$, Prandtl number $P r$, and the velocity ratio parameter $\alpha$ are computed and analyzed. It is found that dual solutions exist for shrinking case and the increase of velocity slip and thermal slip parameters reduces the momentum boundary layer thickness for first solution. Results for the skin friction coefficient, local Nusselt number as well as velocity and temperature profiles are presented for different values of the governing parameters.

\section{REFERENCES}

Bakar, S.A., Arifin, N.M., Ali, F.M., Nazar, R, 2014. "Forced Convection Boundary Layer Slip Flow over a Permeable Plate in a Darcy-Forchheimer Porous Medium," Far East J. Appl. Math. 86(3): 245-261.

Bhattacharyya, K., Mukhopadhyay, S., Layek, G., C., 2011. "Steady Boundary Layer Slip Flow and Heat Transfer over a Flat Porous Plate embedded in a Porous Media," J. Petrol. Sci. Eng. 78:304 - 309.

http://dx.doi.org/10.1016/j.petrol.2011.06.009 
Bhattacharyya, K., Vajravelu, K., Hayat, T., 2013. "Slip Effects on the Parametric Space and the Solution for Boundary Layer Flow of Casson Fluid over a Porous Stretching/Shrinking Sheet," Int. J. Fluid Mech. Res. 40 (6), 482-493.

http://dx.doi.org/10.1615/InterJFluidMechRes.v40.i6.20

Cheng, P., Chang. I-D., 1976. "Buoyancy induced Flows in a Saturated Porous Medium adjacent to Impermeable Horizontal Surfaces," Int. J. Heat Mass Transfer, 19, 1267-1272.

http://dx.doi.org/10.1016/0017-9310(76)90078-8

Cheng, P., 1977. "Similarity Solutions for Mixed Convection from Horizontal Impermeable Surfaces in Saturated Porous Media," Int. J. Heat Mass Transfer, 20, 893-898. http://dx.doi.org/10.1016/0017-9310(77)90059-X

Fang, T., Yao, S., Zhang, J., Aziz, A., 2010. "Viscous Flow over a Shrinking Sheet with a Second Order Slip Flow Model," Commun Nonlinear Sci Numer Simul. 15: 1831 - 1842. http://dx.doi.org/10.1016/j.cnsns.2009.07.017

Hong, J., T., Yamada, Y., Tien, C., L., 1987. "Effect of Non-Darcian and Non-Uniform Porosity on Vertical Plate Natural Convection in Porous Media," Int. J. Heat Mass Transfer 109:356-382. http://dx.doi.org/10.1115/1.3248088

Lok, Y., Y., Ishak, A., Pop, I., 2011. "MHD Stagnation-Point Flow towards a Shrinking Sheet," Int J Numer Meth Head Fluid Flow, 21, 6172.

http://dx.doi.org/10.1108/09615531111095076

Mahapatra, T., R., Nandy, S., K., 2013. "Stability of Dual Solution in Stagnation-Point Flow and Heat Transfer over a Porous Shrinking Sheet with Thermal Radiation," Meccanica, 48, 23-32. http://dx.doi.org/10.1007/s11012-012-9579-5
Mahapatra, T., R., Nandy, S., K., 2013. "Slip Effects on Unsteady Stagnation-Point Flow and Heat Transfer over a Shrinking Sheet," Meccanica, 48, 1599-1606.

http://dx.doi.org/10.1007/s11012-012-9688-1

Mahdy, A., Chamkha, A., J., 2010. "Chemical Reaction and Viscous Dissipation Effects on Darcy- Forchheimer Mixed Convection in a Fluid saturated Porous Media," Int. J. Num. Methods Heat Fluid Flow, 20(8), 924-940.

http://dx.doi.org/10.1108/09615531011081441

Merkin, J., H, Bachok, N., Ishak, A., Pop, I., 2014. “A Comment on the Flow and Heat Transfer past a Permeable Stretching/Shrinking Surface in a Porous Medium: Brinkman Model," Transp. Porous Med., 101, 365371. http://dx.doi.org/10.1007/s11242-013-0249-4

M. Miklavci c, Wang, C. Y., 2006. "Viscous Flow due to a Shrinking Sheet," Int. J. Nonlinear Mech. 64, 283-290.

Mukhopadhyay, S., Layek, G., C., 2009. "Radiation Effect on Forced Convective Flow and Heat Transfer over a Porous Plate in a Porous Medium," Meccanica, 44: 587-597.

http://dx.doi.org/10.1007/s11012-009-9211-5

Mukhopadhyay, S., De, P., R., Bhattacharyya, K., Layek, G., C., 2012. "Forced Convective Flow and Heat Transfer over a Porous Plate in a Darcy-Forchheimer Porous Medium in presence of Radiation," Meccanica, 47,153-161. http://dx.doi.org/10.1007/s11012-011-9423-3

Weidman, P., Kubitschek, D., Davis, A., 2006. "The Effect of Transpiration on Self-Similar Boundary Layer Flow Overmoving Surfaces," International Journal of Engineering Science, 44(11-12), 730-737.

http://dx.doi.org/10.1016/j.ijengsci.2006.04.005 